\title{
Behavior and autonomic nervous system function assessed via heart period measures: The case of hyperarousal in boys with fragile $\mathrm{X}$ syndrome
}

\author{
MARIA L. BOCCIA and JANE E. ROBERTS \\ University of North Carolina, Chapel Hill, North Carolina
}

\begin{abstract}
Physiological responses may inform us about and help us to interpret behavioral responses. For example, hyperarousal may be a source of behavior problems in children with fragile X syndrome (FXS). To evaluate this approach, we examined heart period data in specific contexts in boys with FXS and in normally developing chronological-age-matched boys. Spectral analysis was used to evaluate the parasympathetic and sympathetic nervous systems' contributions to heart period. Boys with FXS had shorter interbeat intervals, lower parasympathetic activity, and similar sympathetic activity. Also, the groups were differentially responsive to experimental challenge. These results have important implications for our understanding of the basic nervous system dysfunction in FXS and for the strategies likely to be effective in terms of pharmacological intervention with these children. These methods can be applied to a variety of contexts and populations, including children who are sensory defensive, socially avoidant, inattentive, or hyperactive.
\end{abstract}

Investigations of individual differences are often enhanced by concomitant consideration of physiological and behavioral responses, and data from multiple sources can often strengthen a behavioral study. Psychobiological perspectives combine these two responses in looking at integrative analyses of both behavior and physiology. Although valuable, collection and coordination of these data often pose significant challenges. Specifically, it is challenging to find a method of data collection that allows integration between two systems, is noninvasive and tolerable to subjects, yet is reliable in terms of signal detection. Although these challenges are present in most studies integrating physiological and behavioral data, they are amplified when working with children who have developmental delays and/or known psychopathologies, particularly when sensory defensiveness is a behavioral characteristic. For example, completing functional magnetic resonance image scanning with unmedicated autistic children would be very challenging, given their difficulties in following directions, remaining still, and tolerating sensory stimulation.

Hyperarousal as a source of behavior problems in children with fragile $\mathrm{X}$ syndrome (FXS) is a case in which

This research was supported by USDEd Grants H023C30081, $\mathrm{H} 023 \mathrm{C} 50034$, and $\mathrm{H} 023 \mathrm{~B} 70035$, by a postdoctoral grant to the second author from the FRAXA Foundation, and by the North Carolina Mental Retardation Research Center, funded by PHS Grant HD03110. Correspondence concerning this article should be addressed to $\mathrm{M}$. L. Boccia, Frank Porter Graham Child Development Center, University of North Carolina at Chapel Hill, 105 Smith Level Road, Chapel Hill, NC 27599-8180 (e-mail: maria_boccia@unc.edu). consideration of physiological responses may inform us about and help us to interpret behavioral responses. Although numerous studies have cited evidence of high levels of behavioral arousal in individuals affected by FXS (Cohen, 1995; Merenstein et al., 1996), there has been only one study of physiological arousal and no study that has integrated behavioral and physiological sources of arousal. In this paper, we will use a study of hyperarousal in boys with FXS as a model of integrating multiple systems in order to study physiological arousal and its relation to behavioral problems.

\section{Fragile X Syndrome}

FXS is the primary known cause for hereditary mental retardation (Hagerman \& Cronister, 1996). Although it may be found in both males and females, males are more severely affected. Approximately $85 \%$ of males with FXS are developmentally delayed, and virtually all have learning difficulties. Behavioral difficulties often constitute the most significant barrier to successful functioning and are cited by parents and educators as their primary concern (Hatton \& Bailey, 1997). Common behavioral difficulties resemble those seen in etiology-specific groups noted for arousal regulation difficulties, including ADHD (e.g., being impulsive, fidgety, inattentive, distractible, emotionally labile, and aggressive and showing excessive motor activity) and autism (e.g., showing gaze aversion, sensory integration problems, attention difficulties, social skill deficits, self-abuse, aggression, and motor stereotypies; Baumgardner, Reiss, Freund, \& Abrams, 1995). These behaviors are most frequently observed in transitions, interpersonal exchanges, and high- 
demand performance activities, which has led to the hypothesis that these behaviors result from physiological hyperarousal (Cohen, 1995).

Although clinical work suggests an excessive initiation of arousal, indicating that the sympathetic nervous system may be primarily responsible for arousal regulation difficulties in FXS, this is not necessarily the case. Porges (1996) has found that individuals with low vagal (parasympathetic) tone have difficulty regulating their behavior, whereas those with average or high vagal tone do not have these difficulties. In typically developing children, this may be related to developmental changes in autonomic nervous system (ANS) functioning parallel to behavioral development. As behavior matures and shifts from reflexes and homeostatic functions to behaviors that are more cognitively mediated, there is a subordination and integration of primary reflexive behavior into more complex voluntary behavior. This, Porges (1996) suggests, corresponds to a change of balance in the nervous system from more sympathetic to more parasympathetic input. In children with developmental disabilities, this process may be incomplete, and arousal regulation difficulties may be due to inefficient vagal regulation.

\section{Autonomic Nervous System and Behavior}

Within the central nervous system, the limbic system serves to integrate sensory input systems with output systems. This system, which includes a variety of subcortical structures, coordinates input from vision, audition, taste, smell, and touch, directs these inputs to higher cortical centers for integration and analysis, and provides pathways through which these higher cortical centers can influence output systems, such as endocrine or motor responses. Thus, the ANS, of which the limbic system is a part, is critical for the regulation of arousal (Boop, GarciaRill, Dykman, \& Skinner, 1994; Porges, 1992; see Fox \& Fitzgerald, 1990, and Szymusiak, 1995, for reviews). Many of the physiological response systems, therefore, that are of interest to biobehavioral researchers are controlled by this compartment of the nervous system.

Peripheral indicators of ANS activity, such as heart rate or hormones, can be used to index these functions, and information about ANS function can inform our understanding of behavior. One behavior class that may profit from such an analysis, for example, is stereotypies (Nijhof, Joha, \& Pekelharing, 1998; Soussignan \& Koch, 1985). Stereotypies are idiosyncratic behaviors, often repetitive, generally involving some kind of motor activity that apparently serves no immediate identifiable function. Examples can include head wagging, hand flapping, pacing, and bizarre postures. One theoretical perspective postulates that these behaviors are displayed during times of excessive stimulation and hyperarousal and serve to calm the individual down. The stereotypy, in this perspective, lowers levels of arousal, thereby regulating the intensity of the stimulation for the individual. An alternative perspective suggests that this behavior serves to stimulate an individual when that individual is bored- that is, experiencing low levels of stimulation and, therefore, low levels of arousal. The stereotypy, in this perspective, increases the level of arousal by providing stimulation in an otherwise unstimulating environment. Clearly, these two explanations are diametrically opposed to one another. Measurement of a physiological indicator of arousal might clarify which of these explanations applies in a particular case.

\section{Measurement of Heart Period}

Heart period measures have been used extensively in a variety of contexts as an index of ANS function in studies of individual differences and behavior-physiology interactions (Porges \& Byrne, 1992). Statistical analysis of the variability in interbeat intervals (IBIs) permits us to separate the sympathetic and parasympathetic contributions to variability. In fact, multiple indices are available for measuring these parameters, and data can be collected noninvasively, which makes this approach to physiological measurement very appealing in the context of dealing with humans in general and children in particular.

The heart period may be represented as an IBI or cycle length, which is the time between successive $\mathrm{R}$-waves. Hainsworth (1995) demonstrated that the relationship between the vagal nerve firing rate and the IBI and that between the heart rate and the IBI are quite different. In particular, the IBI is related to the vagal firing rate in a clear, simple linear relationship. That is, increases in IBI directly correspond to equivalent increases in vagal firing rate across the entire range of intervals examined. The implication is that one may infer change in vagal firing rate from change in IBI similarly across its entire range of variability.

\section{Time Series Analysis of Heart Period Data}

Heart period data are given in a form known as time series (see Porges \& Bohrer, 1990, for a review). A time series is any data series that is collected over consecutive points in time. Often, time series have characteristics, such as repeating patterns, that transcend typical simple descriptive statistics, such as mean or standard deviation. Traditional descriptive statistics of time series are not able to evaluate the unique differences in the patterning of the data in time series. Specialized statistical analyses have been developed to permit us to describe those differences in patterning, which are called time series analyses. Spectral analysis, one form of time series analysis, decomposes time series into its constituent components. In particular, it analyzes the component sine waves that describe the patterns in the data. The spectral analysis represents these sine waves' wavelengths in terms of their frequency and represents their amplitude as power. Each frequency in the spectral analysis represents a different sine wave, and the power of that frequency represents the contribution of that component to the variability of the time series. These techniques were originally designed to analyze complex nonorganic signals but have been adapted for use in analyzing heart period data (Kamath \& Fallen, 
1993). There are two major peaks typically found in spectra of heart period - one low-frequency peak and one high-frequency peak. The actual locations of these peaks will vary with the age of the individual being studied and with a variety of other parameters. For children, however, these peaks typically reside in the 0.04-0.24 range for the low-frequency band and in the 0.24-1.04 range for the high-frequency band (Porges \& Byrne, 1992).

The spectral peaks in these frequency bands represent variability in the heart signal produced by parasympathetic (specifically vagal) and sympathetic (specifically $\beta$-sympathetic) activity. Although other parameters associated with heart signal ( $\mathrm{R}-\mathrm{T}$ transit time, blood pressure variation, etc.) contain information about ANS inputs, these data are useful in that they represent relatively noninvasive ways to get this information. Drug blockade studies have demonstrated how the parasympathetic and sympathetic nervous systems contribute to these peaks (Akselrod, 1981; Akselrod et al., 1985; Pomeranz et al., 1985). Parasympathetic blockade results in total elimination of the high-frequency peak and partial elimination of the low-frequency peak. This suggests that the highfrequency peak is produced exclusively, or nearly exclusively, by the parasympathetic nervous system input into the heart signal. Total autonomic blockade, which blocks both sympathetic and parasympathetic contributions, results in elimination of the remaining component of the low-frequency band. This indicates that the low-frequency peak includes both sympathetic and parasympathetic components. It has been suggested, therefore, that one can compute estimates of parasympathetic and sympathetic contributions to the heart period variability by examining high-frequency peak power as representing the contribution of the parasympathetic nervous system (Akselrod, 1995). A ratio of the low-frequency band to the highfrequency band can be calculated as representing the contribution of the sympathetic nervous system to the spectrum, especially when the focus is sympathovagal balance (Akselrod, 1995).

\section{Heart Period and Arousal \\ in Boys With Fragile X Syndrome}

In the experiment described below, physiological indices of arousal involving heart period were investigated, comparing boys with FXS with typically developing peers. The heart period measures included mean heart period, or IBI, vagal tone estimate, and sympathetic tone estimate. These variables were examined while the children participated in an experimental protocol of alternating passive and active forms of behavioral and cognitive demand.

We expected to find that boys with FXS would display a shorter and less variable IBI than boys without FXS and that boys with FXS would exhibit significantly higher sympathetic tone estimates and lower vagal tone estimates than boys without FXS. We also expected that there would be significantly different patterns of heart period measures between the active and the passive phases of the experiment within the two groups of boys.

\section{METHOD}

We have taken advantage of a simple system for collecting data from individuals for whom it would otherwise be difficult or impossible to participate in research. In particular; children with disabilities who are tactile defensive or otherwise resistant to the placement of electrodes and other intrusive procedures, such as collection of blood samples or utilization of blood pressure cuffs, can participate in this research. By utilizing a system for collecting IBI data, we can access information about both sympathetic and parasympathetic activity, using methods that these children will tolerate. Specifically, we have begun using Mini-Logger 2000 systems produced by Minimitter, Inc. (Sunriver, OR; Mini-Logger 2000, 1994) for this purpose. This system uses Polar chest belts to detect R-waves. The belt is fitted to the child's chest at the base of the sternum and is secured with a snap. No glue or electrolyte is required, because the child's own perspiration establishes the contact points. This radiotelemetry system eliminates the need for traditional electrodes, which must be glued in place and, hence, are potentially aversive to young children with and without disabilities. The Mini-Logger 2000 is a small, portable receiver set to the frequency of the Polar belt transmitter that collects and stores IBIs into files subsequently downloaded to PC computers for analysis. Minimitter software converts the logger data to an ASCII file that can be imported into other programs for editing and analysis.

To verify that the Mini-Logger 2000 and Polar chest belt were accurate and reliable instruments for collecting heart period data, we collaborated with another laboratory to evaluate this, simultaneously using both Porges' data collection system (described in Calkins, 1997) and the Mini-Logger 2000 with the Polar chest belt with data from 1 subject (we thank Susan Calkins for assisting us with this effort). Data from a 10-min sample of IBIs from both systems were compared, and the results indicted that the two systems produced highly concordant results. The mean difference between the IBI values generated by the two systems was $3 \mathrm{msec}$.

Once the heart period data are collected and converted to an ASCII file, they can be imported into other software, such as MXEdit (MxEdit 2.01, 1989). After collection of the data, we edit the files for errors, and analyze them via spectral analysis. This method, provided as an analysis component of MXEdit, uses Porges' algorithms for spectral analysis. This method detrends and filters the IBI data and permits one to resample the data at an appropriate rate for subsequent analysis (i.e., twice the mean heart period for the subject) and to determine the desired frequency bands to analyze. A moving polynomial analysis procedure is used to account for nonstationarity. Output is mean IBI and the natural logarithm of the heart period variance in the frequency band specified. The results can be used to calculate the relative contribution of the sympathetic and parasympathetic systems. For children in the range of ages that we study, the 0.24-1.04 Hertz band includes the highand mid-frequency peaks (representing parasympathetic input into the IBI pattern), whereas the 0.04-0.24 Hertz band includes the low-frequency peak) and includes both sympathetic and parasympathetic input (Porges \& Byrne, 1992). An estimate of the relative contribution of sympathetic input into the IBI pattern can be derived by computing the ratio of the power in the 0.04-0.24 Hertz range to the power in the $0.24-1.04$ Hertz range (Akselrod, 1995).

The Observer (Noldus Information Technology, 1996) is a commercially available software package designed to facilitate the direct, on-line collection of behavioral observations, either in a live situation or from videotape. We have found it to be an excellent system for our applications im terms of reliability, flexibility, and technical support. This program provides a general purpose system for the collection of user-customized behavioral observation data. As such, it provides sufficient flexibility to be as complicated or as simple as required by the demands of the particular research protocol.

The DOS version of The Observer (Noldus Information Technology, 1991) has an additional feature of being able to read exter- 
Table 1

Mean (and Standard Error of the Mean)

Interbeat Interval, Vagal Tone, and Sympathetic

Tone Estimates for Boys With and Without FXS

\begin{tabular}{|c|c|c|c|c|c|c|c|c|c|c|c|c|}
\hline \multirow[b]{4}{*}{ Phase } & \multicolumn{12}{|c|}{ Heart Period Variable } \\
\hline & \multicolumn{4}{|c|}{$\begin{array}{c}\text { Interbeat Interval } \\
\text { (msec) }\end{array}$} & \multicolumn{4}{|c|}{$\begin{array}{c}\text { Sympathetic } \\
\text { Tone Estimate }\end{array}$} & \multicolumn{4}{|c|}{$\begin{array}{c}\text { Vagal } \\
\text { Tone Estimate }\end{array}$} \\
\hline & \multicolumn{2}{|c|}{ FXS } & \multicolumn{2}{|c|}{ Typical } & \multicolumn{2}{|c|}{ FXS } & \multicolumn{2}{|c|}{ Typical } & \multicolumn{2}{|c|}{ FXS } & \multicolumn{2}{|c|}{ Typical } \\
\hline & $M$ & $S E M$ & $M$ & $S E M$ & $M$ & $S E M$ & $M$ & $S E M$ & $M$ & $S E M$ & $M$ & $S E M$ \\
\hline & 54 & 89 & 58 & 87 & 3 & 5 & 0.47 & 1 & 4.75 & 1.47 & 5.71 & 1.38 \\
\hline & 546 & 09 & 576 & 76 & 0.47 & 0.10 & 0.48 & 0.10 & 4.90 & 1.45 & 5.66 & 0.9 \\
\hline sk 2 & 543 & 89 & 572 & 89 & 0.46 & 0.15 & 0.46 & 0.13 & 4.78 & 1.41 & 5.68 & 1.33 \\
\hline & 544 & 93 & 580 & 76 & 0.49 & 0.10 & 0.48 & 0.12 & 4.83 & 1.27 & 5.77 & 1.09 \\
\hline assive task 3 & 543 & 86 & 575 & 93 & 0.45 & 0.17 & 0.45 & 0.11 & 4.95 & 1.40 & 5.68 & 14 \\
\hline
\end{tabular}

nal files containing other types of data. Thus, one can use The $\mathrm{Ob}-$ server to integrate behavioral observations collected with the system and continuous physiological data collected simultaneously on another system. Because The Observer allows one to use files generated from other systems, this provides the greatest flexibility in terms of choice of physiological measure. One is not restricted to what Noldus has implemented for physiological assessment. For example, we have used this system both to integrate heart rate and behavior in our research with monkey models of bereavement and to examine the effect of different emotional expressions on hemispheric lateralization via EEG (Boccia, Davis-Goldman, Noldus, O'Leary, \& Teale, 1995). We describe here the application of this system to the study of hyperarousal in boys with FXS, in which we use multiple heart rate measures, including raw heart period, as well as vagal tone and other measures.

\section{Subjects}

The boys with FXS were drawn from the Carolina Fragile X Project, which spans a three-state area, including North Carolina, South Carolina, and Virginia (Bailey, Hatton, \& Skinner, 1998). Children were excluded if they were taking medication with documented cardiovascular effects or if there was documentation of a known history of cardiovascular disease. Sixty-two boys, half with and half without FXS, were studied. We discuss here a subset of 40 of these children, for whom data analysis is complete, including 20 boys with FXS (mean chronological age $[\mathrm{CA}]=4.03$ ) and 20 typically developing boys (mean $\mathrm{CA}=4.05$ ).

\section{Procedure}

The children participated in a 25 -min experimental session that comprised five phases lasting 5 min per phase. The first, third, and fifth phases were passive tasks (watching a video), and the second and fourth phases were active (completing cognitive tasks) phases. Thus, the child experienced alternating passive and active task situations in an $\mathrm{A}-\mathrm{B}-\mathrm{A}-\mathrm{B}-\mathrm{A}$ pattern. Each phase was approximately $5 \mathrm{~min}$ in duration. The cognitive tasks were adaptations of subtests from the Stanford-Binet IV and included a verbal (vocabulary) and nonverbal (pattern analysis) task. Data on the mental age of the children with FXS and the chronological age of the typical children were used to determine where to begin each test to provide ageappropriate cognitive challenges to each child. Videotapes of the experimental sessions were coded, using The Observer.

The Observer was used to analyze experimental phases concomitant with all variables related to heart rate. This was accomplished by synchronizing the IBI data and the experimental phases coded in an Observer data file. The Mini-Logger receiver includes an event code marker that, when activated, places a marker code in the data stream that can later be identified. During data collection, a marker is placed in the Mini-Logger receiver file within visual range of the video camera, which is recording the experimental session. The Observer scoring system includes a code for marker event, which is then used to synchronize the heart period time series with the observational data. This permits synchronization of the heart period time series and observational data at the resolution of The Observer software, which is $0.01 \mathrm{sec}$.

The Mini-Logger 2000, as described above, was used to collect the heart period data. The heart period data were analyzed using the software program MxEdit (MxEdit 2.01, 1989). The data files of successive heart periods for each subject were edited to remove artifacts and to correct errors and were analyzed for mean IBI, sympathetic tone estimate, and vagal tone estimate. Briefly, following editing, data analysis includes ( 1 ) converting the heart periods to constant sampling rates by resampling the data at approximately twice the duration of the IBI in this data set (in this case, 250-msec intervals), (2) detrending the resampling data with a 21-point moving cubic polynomial to control for nonstationarities in the data, (3) processing the detrended data with a digital bandpass filter to remove sources of variance outside the frequency band chosen (e.g., 0.24-1.24 for vagal tone for children in this age range), and (4) computing the natural logarithm of the bandpassed variance, which permits the data to be subjected to parametric statistical analyses. All the subsequent analyses were completed using SPSS for Windows 7.5 (SPSS, 1998). A two (group) $\times$ five (phase) repeated measures analysis of covariance, with $\mathrm{CA}$ as a covariate, was performed on the data.

\section{RESULTS}

The boys with FXS differed from the typically developing boys in a number of ways, including shorter heart period (i.e., faster heart rate), a less active parasympathetic system (lower vagal tone), and different patterns of arousal across the experimental phases. Values of dependent measures for the two groups are presented in Table 1. These values are raw scores, unadjusted for age of the children.

The boys with FXS displayed a significantly higher heart rate, reflected in shorter IBI values $[F(1,36)=4.438$, $p<.05]$. There was no significant main effect of group for the sympathetic tone estimate. There was, however, a significant difference in vagal tone between the boys with FXS and the typically developing boys $[F(1,36)=$ $7.383, p<.01]$. The boys with FXS had significantly 
lower vagal tone estimates than did the typically developing boys.

Finally, for sympathetic tone estimates among the boys with FXS, a significant effect of phase was found $[F(1,17)=3.629, p<.01]$. Post hoc analyses indicated that the first baseline differed from the first cognitive task and the intermediate baseline from the second cognitive task. For the typically developing boys, no effect of phase was found for the sympathetic tone estimates.

For vagal tone estimates, there was no significant effect of phase for the boys with FXS. For the typically developing boys, a significant effect of phase was found $[F(1,17)=5.788, p<.001]$. Post hoc analyses indicated that each cognitive task differed significantly from the adjacent baselines.

\section{CONCLUSIONS}

This study models the integration of multiple methods in the study of physiological hyperarousal as a source of behavior problems in FXS. The results of this study support the hypothesis that boys with FXS exhibit levels and patterns of physiological arousal that differ from typically developing, CA-matched peers. These differences in physiological arousal include shorter heart period (i.e., faster heart rate), a less active parasympathetic system (vagal tone), and different patterns of arousal regulation across the experimental phases. This suggests that the higher level of behavioral and physiological arousal in these children may be due more to reduced parasympathetic activity than to enhanced sympathetic activity.

Although the differences in heart rate and vagal tone are statistically significant, they have no medical significance in themselves. However, these differences in heart period do have theoretical and clinical relevance. The observation of higher levels of arousal owing to reduced parasympathetic activity extends the work completed by Porges and his colleagues. Specifically, they found that individuals with low and unresponsive vagal tone had difficulty with behavior regulation. This observation led Porges to develop the polyvagal theory. The polyvagal theory emphasizes the functional difference between vagal fibers that provide a neurophysiological substrate for behavioral responses (Porges, 1996). Since these investigators focused on typically developing children, parallel findings in boys with FXS in this study extend their theory to include a sample of children with developmental delays of genetic etiology. Therefore, the behavioral challenges evident in boys with FXS may be a reflection of inadequate physiological self-regulation that may be associated with particular brain nuclei, particularly in the limbic system. Clinically, the findings in support of the hyperarousal theory in FXS support consideration of environmental modifications (i.e., minimizing high-demand situations) and psychopharmacologic treatment.

In conclusion, the study of physiology in relation to behavior allows us to draw implications about the neurophysiological basis of problem behavior that would not be possible by studying behavior in isolation. These types of studies are increasingly feasible, given recent methodological advances in technology and multisystems integration that make data collection and analysis more feasible. The nonaversive nature of many of the newest systems means that these techniques can be used with subjects that have been traditionally excluded from this type of psychophysiological research because of ethical concerns or because of technical difficulties in getting participation from individuals who have developmental disabilities and may exhibit tactile defensiveness. Furthermore, the ease of analysis now possible because of the availability of software such as The Observer by Noldus Information Technology makes the integration of behavior and physiology cost effective. The experiment described here with children with FXS suggests that significant advances in understanding the biological correlates of behavior, especially in populations with developmental disabilities, is now possible with these new technologies.

\section{REFERENCES}

AKSELROD, S. (1981). Power spectrum analysis of heart rate fluctuation: A quantitative probe of beat-to-beat cardiovascular control. Science, 213, 220-222.

AKSELROD, S. (1995). Components of heart rate variability: Basic studies. In M. Malik \& A. J. Camm (Eds.), Heart rate variability (pp. 147163). Armonk, NY: Futura.

akselrod, S., Gordon, D., Madwed, J. B., Snidman, N. C., ShanNoN, D. C., \& CoHeN, R. J. (1985). Hemodynamic regulation: Investigation by spectral analysis. American Journal of Physiology, 249, 867-875.

Bailey, D. B., Hatton, D. D., \& Skinner, M. (1998). Early developmental trajectories of males with fragile $\mathrm{X}$ syndrome. American Journal on Mental Retardation, 103, 29-39.

Baumgardner, T., Reiss, A. L., Freund, L. S., \& Abrams, M. T. (1995). Specifications of the neurobehavioral associations in males with fragile X syndrome. Pediatrics, 95, 744-752.

Boccia, M. L., Davis-Goldman, B., Noldus, L. P. J. J., O'Leary, M., \& Teale, P. (1995). The integration of physiology (heart rate and EEG) with behavior collected with a computer based observational system. In E. Alleva, A. Fasolo, H.-P. Lipp, L. Nadel, \& L. Ricceri (Eds.), Behavioral brain research in naturalistic \& semi-naturalistic settings (NATO ASI Series D: Behavioural and Social Sciences ed., Vol. 82, p. 440). London: Kluwer Academic Publishers in cooperation with NATO Scientific Affairs Division.

Boop, F. A., Garcia-Rill, E., Dykman, R., \& Skinner, R. D. (1994). The $\mathrm{P}_{1}$ : Insights into attention and arousal. Pediatric Neurosurgery, 20, 57-62.

CALKINS, S. D. (1997). Cardiac vagal tone indices of temperamental reactivity and behavioral regulation in young children. Developmental Psychobiology, 31, 125-135

COHEN, I. L. (1995). A theoretical analysis of the role of hyperarousal in the learning and behavior of fragile X males. Mental Retardation \& Developmental Disabilities Research Reviews, 1, 286-291.

Fox, N. A., \& FitzGerald, H. E. (1990). Autonomic function in infancy. Merrill-Palmer Quarterly, 36, 27-51.

Hagerman, R. J., \& Cronister, A. (EDS.) (1996). Fragile X syndrome: Diagnosis, treatment, and research (2nd ed.). Baltimore: Johns Hopkins University Press.

HAINSWORTH, R. (1995). The control and physiological importance of heart rate. In M. Malik \& A. J. Camm (Eds.), Heart rate variability (pp. 3-19). Armonk, NY: Futura.

HATTON, D. D., \& BaILEY, D. B. (1997). Early intervention for young boys with fragile $X$ syndrome. National Fragile $X$ Advocate, 2(3), 14-16. 
Kamath, M. V., \& Fallen, E. L. (1993). Power spectral analysis of heart rate variability: A noninvasive signature of cardiac autonomic function. Critical Reviews in Biomedical Engineering, 21, 245-311.

Merenstein, S. A., Sobesky, W. E., Taylor, A. K., Riddle, J. E., TRAN, H. X., \& HAGERMAN, R. J. (1996). Molecular-clinical correlations in males with an expanded FMR1 mutation. American Journal of Medical Genetics, 64, 388-394.

Mini-LogGer 2000 [Computer software] (1994). Sunriver, OR: MiniMitter $\mathrm{Co}$.

MxEDIT 2.01 [Computer software] (1989). Bethesda, MD. DeltaBiometrics.

Nijhof, G., Joha, D., \& Pekelharing, J. (1998). Aspects of stereotypic behaviour among autistic persons: A study of the literature. British Journal of Developmental Disabilities, 44, 3-13.

Noldus, L. P. J. J. (1991). The Observer: A software system for collection and analysis of observational data. Behavior Research Methods, Instruments, \& Computers, 23, 415-429.

Noldus InFoRMATION TECHNOLOGY (1991). The Observer system for collection and analysis of observational data: Version 3.0. Wageningen, The Netherlands: Author.

Noldus INFORMATION TECHNOLOGY (1996). The Observer system for collection and analysis of observational data: Version 3.0 for Windows. Wageningen, The Netherlands: Author.

Pomeranz, B., Macaulay, R. J. B., Caudill, M. A., Kutz, I., Adam, D., Gordon, D., Kilborn, K. M., Barger, A. C., Shannon, D. C.,
Cohen, R. J., \& Benson, H. (1985). Assessment of autonomic function in humans by heart rate spectral analysis. American Journal of Physiology, 248, H151-H153.

PORGES, S. W. (1992). Vagal tone: A physiologic marker of stress vulnerability. Pediatrics, 90, 498-504.

Porges, S. W. (1996). Physiological regulation in high-risk infants: A model for assessment and potential intervention. Development \& Psychopathology, 8, 43-58.

PORGES, S. W., \& BoHRER, R. E. (1990). The analysis of periodic processes in psychophysiological research. In J. T. Cacioppo \& L. G. Tassinary (Eds.), Principles of psychophysiology: Physical, social and inferential elements (pp. 708-753). New York: Cambridge University Press.

Porges, S. W., \& BYrNe, E. A. (1992). Research methods for measurement of heart rate and respiration. Biological Psychology, 34, 93-130.

Soussignan, R., \& КосH, P. (1985). Rhythmical stereotypies (leg swinging) associated with reductions in heart rate in normal school children. Biological Psychology, 21, 161-167.

SPSS (1998). SPSS for Windows, release 8.0. Chicago: Author.

SzymuSIAK, R. (1995). Magnocellular nuclei of the basal forebrain: Substrates of sleep and arousal regulation. Sleep, 18, 478-500.

(Manuscript received December 18, 1998; revision accepted for publication October 3, 1999.) 